\title{
A KNOWLEDGE SHARING FRAMEWORK FOR HOMELAND SECURITY MODELING AND SIMULATION
}

\author{
Sanjay Jain \\ Charles W. Hutchings \\ The George Washington University \\ U.S. Department of Homeland Security \\ 2201 G Street NW, Suite 415 \\ Washington, DC 20052, USA \\ Science \& Technology Directorate/TSD \\ Washington, DC 20528, USA \\ Y. Tina Lee \\ Charles R. McLean \\ National Institute of Standards and Technology \\ 100 Bureau Drive, MS826 \\ Gaithersburg, MD 20899, USA
}

\begin{abstract}
Modeling and simulation $(\mathrm{M} \& \mathrm{~S})$ tools and capabilities can enable understanding of the complex nature of systems in various homeland security domains. A coordinated effort across government, industry, and academia would advance capabilities in this important area. Initiating such an effort requires establishing a common understanding or framework that shares the current knowledge in M\&S. This paper describes an outline for a knowledge-sharing framework that could be developed and maintained by the community interested in tracking and guiding coordinated development efforts in homeland security modeling, simulation, and analysis (MSA).
\end{abstract}

\section{INTRODUCTION}

Modeling, simulation, and analysis (MSA) techniques and tools can be useful for problem solving and decision making across a range of homeland security application domains, including social behavior, natural phenomena, environment, economy and finance, organization, critical infrastructure, and other systems, equipment and tools (McLean, Jain, \& Lee 2008). The use of sound MSA capabilities across all the domains would enhance decision making and response to natural and man-made threats and hazards.

MSA efforts must be coordinated to avoid duplication of effort and to efficiently identify gaps to advance capabilities (Adam 2008). Currently, modeling and simulation tools are being developed in an ad hoc manner within DHS to address specific problems identified by homeland security "customers" such as first responders (Hutchings 2009). A number of U.S. Department of Homeland Security (DHS) sponsored MSA developments are being carried out with industry and university partners, while industry and academic researchers are also developing MSA tools on their own based on their technical innovations and perceived needs.

A DHS Critical Infrastructure (CI) Modeling \& Simulation Workshop (Adam 2008) recommended to:

- Develop and regularly update a master compendium of available models and related research from labs, academia, and industry. 
- Conduct research to define the model attributes and characteristics that must be included for the entries in the compendium.

- Develop methods for communicating the scope and limitations of models in a manner as transparent as possible.

A knowledge sharing framework for homeland security MSA could enable a compendium of models and serve as a catalyst to promote dialogue and a common understanding of analytical tools and techniques. A framework is a classification schema that defines a set of categories into which various concepts or artifacts can be arranged. The proposed framework is used to categorize knowledge assets relevant to homeland security modeling and simulation technical areas. It can support both research and development efforts for MSA by identifying what is available and the remaining gaps. It may be noted that the proposed framework does not aim to describe an enterprise architecture and hence would not fit the schemes such as those presented in Zachman (2008) or Department of Defense Architectural Framework (DoD DCIO 2010).

The knowledge sharing framework will be useful for facilitating sharing of MSA information. A critical step in a coordinated effort is the development of a common understanding of key concepts and the utilization of terminology that is or should be standard within the associated technical and user communities. Technical and user communities should be identified to define and reach consensus on MSA capabilities, assess the current status of these capabilities, and identify gaps that should be addressed to prioritize and meet current and future challenges in their areas of interest.

The technical and user communities may not be aligned with the domain classification from McLean, Jain, and Lee (2008) identified above. In general, such communities are expected to be focused on a subset of the domain since the domains have been defined at a fairly high level. For example, there is a technical community focused on hazardous material release that is a subset of the natural phenomenon domain. Some communities may overlap across the domains. For example, the community involved in modeling human behavior may overlap the social behavior and organizational domains. The technical interest groups for sharing of MSA knowledge hence have been defined differently than the domains identified above though there is some overlap. Currently identified areas that may have associated technical user communities include hazardous material release (HMR), healthcare systems, critical infrastructure (CI), incident command, natural disasters, and traffic, transportation, and evacuations.

This paper describes a structure for the knowledge sharing framework that can be used as a common structure across different technical user communities. Technical interest groups are being identified that will guide the task of populating the information and decide the research and development agenda for their area of interest. Examples from different technical areas are provided to help understand the application of the framework.

\subsection{MSA Development Process}

The knowledge sharing framework should support MSA development efforts. While the detailed description of the development process is outside the scope of this paper, a brief overview is provided to show how the framework may support the MSA development in the next subsection. A structured process should be followed to determine the analytical capabilities appropriate to the problem. Rational problem solving and decision support typically proceed in phases. These might include identification of a problem situation, problem formulation, creation of an evaluation model, analysis, and results or recommendations. The focus of this paper is on the analysis supported by modeling and simulation (M\&S) capabilities. It should be noted that only a subset of the problems may be addressed using M\&S while other techniques such as analytical modeling or experimentation may be used as well.

A guideline for the MSA development process should be defined to allow a common approach. The approach should be applicable across domains of interest to homeland security and should reside as a standard outside the knowledge sharing framework for MSA.

The MSA development process may include the following major phases: 
- Problem identification

- Requirements specification

- Model development

- Model evaluation

- Model application

\subsection{Overview of the Framework}

The knowledge sharing framework would support MSA research and development efforts as shown in figure 1. It would define the needs and requirements based on input from the subject matter experts, researchers and users and ensure the best use of constrained resources that are required for developing useful MSA tools. Currently available tools, ongoing projects, and facilities would be identified to avoid duplication of efforts. Best practices would be shared to allow all to learn from experiences of others. Known research, development, and implementation issues would be captured and shared. Current and needed standards would be identified to ensure interoperability of developed MSA tools.

\begin{tabular}{|c|c|c|}
\hline Research & $\begin{array}{c}\text { Framework for Modeling \& } \\
\text { Simulation }\end{array}$ & Development \\
\hline $\begin{array}{l}\text { Subject matter experts \& } \\
\text { researchers role }\end{array}$ & Knowledge Assets & $\begin{array}{l}\text { MSA development process } \\
\text { phases receiving guidance }\end{array}$ \\
\hline \multirow{5}{*}{$\begin{array}{l}\text { IDENTIFY and provide } \\
\text { assets }\end{array}$} & Scope & \multirow{2}{*}{ Problem Identification } \\
\hline & Needs & \\
\hline & Requirements Specification & Requirements Specification \\
\hline & $\begin{array}{l}\text { Resources: } \\
\text { - Projects, Facilities \& Capabilities } \\
\text { - Tools Summary } \\
\text { - Relevant Standards \& Guidelines } \\
\text { - Data Sources \& Formats }\end{array}$ & \multirow{3}{*}{$\begin{array}{l}\text { Model Development } \\
\text { Model Evaluation } \\
\text { Model Application }\end{array}$} \\
\hline & \multirow{2}{*}{$\begin{array}{l}\text { Discussions \& Recommendations: } \\
\text { - Best Practices } \\
\text { - Limitations, Cautions \& Warnings } \\
\text { - Research, Development, Standards } \\
\text { \& Implementation Issues }\end{array}$} & \\
\hline $\begin{array}{c}\text { DEFINE research agenda; } \\
\text { CONDUCT Research and } \\
\text { Development }\end{array}$ & & \\
\hline DEVELOP assets & $\begin{array}{l}\text { Framework Reference Materials } \\
\text { (Glossary, Research Literature Refer- } \\
\text { ences, etc.) }\end{array}$ & All \\
\hline
\end{tabular}

Figure 1. Overview of Knowledge Sharing Framework for Homeland Security Modeling and Simulation technical areas and its linkages with Research and Development 


\subsection{Organization of the Paper}

This paper describes the assets that would constitute the knowledge sharing framework for homeland security MSA. It is proposed that the framework be utilized to capture knowledge under the guidance of technical interest groups comprising the stakeholders devoted to areas relevant to homeland security MSA. Knowledge and insights captured in the framework could be available to the community in a report or on-line using mechanisms such as a secure portal or Wiki. The following sections respectively describe the knowledge assets in the framework in the sequence shown in figure 1. The last section concludes the paper.

\section{SCOPE OF THE FRAMEWORK}

A knowledge sharing framework for a coherent area of homeland security MSA needs to include the subareas that are considered relevant by the corresponding technical interest group. For example, for the CI area the scope should include cross-cutting concerns with modeling, simulation, and analysis in all eighteen CI areas which are currently identified (USDHS 2010a). The framework would incorporate information across all phases of infrastructure lifecycle including planning, designing, building, operating, and decommissioning. As CI MSA capabilities evolve, separate knowledge sharing frameworks may be needed for each CI sector to reflect sector-specific needs and concerns. Similarly, for the HMR area the scope may include modeling and simulation of the release chemical, biological, nuclear, and radiological agents, as well as natural occurring releases such as volcanoes and wild fires. Associated models and simulations may involve the release of materials into the atmosphere, within buildings and other structures including heating ventilation and air conditioning (HVAC) systems, bodies of water and watershed systems, as well as ground contamination.

The scope for healthcare systems technical area should include simulation and modeling activities that support analysis, planning, and training needs for the healthcare institutions, epidemics, and other healthcare-related emergencies. Simulation models may be used to understand healthcare systems, interdependencies with other systems, their vulnerabilities, and the impact of emergency incidents on the population and healthcare community.

For all the technical areas identified, the MSA tools may be used for all the application types defined in McLean, Jain, \& Lee (2008) to inform analysis and decision support, planning and operations, systems engineering and acquisition, and training, exercises and performance measurement.

\section{NEEDS ANALYSIS OVERVIEW}

Stakeholders at all levels need analytical tools and methods to address problems and support decision making. The purpose of this section of the framework would be to capture problem situations that are commonly encountered by stakeholders across the identified technical area and how M\&S capabilities are used to support the problem solving/decision making processes at more than one level. For example in the case of CI, given a major hurricane and potential impacts, the Federal Emergency Management Administration (FEMA) will have a set of concerns, state and local officials in potentially affected areas another set of concerns, and power generating facilities still other concerns. This will promote a common understanding of analytical issues from multiple perspectives and support the development and sharing of analytical tools and M\&S capabilities across stakeholder organizations.

This section of the framework will identify, document, and catalogue problems that are most likely to be faced in the particular technical area. It will help define the relevant analytical needs and determine the right decision-relevant questions that need to be addressed, establishing the purpose and objectives for MSA capabilities. Examples of questions that may be captured as needs for using MSA for CI include:

- How will the impact of a natural disaster such as hurricane, tornado, or violent lightning storms striking parts of the power grid affect outage management? 
- What will be the impact of disruption in one critical infrastructure on other co-located or connected critical infrastructure systems? For example, how will disruption in the power grid affect the water delivery infrastructure for a city?

A recent study has identified research questions for complex network questions (Brase and Brown 2009). Since CI systems are networked systems, the identified questions contribute to needs analysis as indicated by examples below.

- Can we use, understand, and quantify the efficacy of new security approaches for computer networks?

- Can we improve the design of computer or communication networks to be more robust against partial failures or intentional attacks?

- Can we understand how populations will respond to the availability of new energy sources, or to changes in energy policy?

Similarly for the HMR area, on the occurrence of a release, officials want to know - What is the hazard? Where is it going? Who is at risk? How do we respond? Specific questions that may be answered using simulation include the following.

- What is the forecasted transport direction of the plume and what areas may be under hazard?

- What are the estimated potential damages, casualties, illnesses, and fatalities?

- What are the estimated emergency assistance requirements?

- What are the areas where buildings, land, agricultural crops, bodies of water, and other man-made or natural resources are or will be contaminated?

\section{MSA REQUIREMENTS SPECIFICATIONS}

The high level needs analysis should form the basis for requirements specifications for the technical area. To address the analytical needs, each MSA capability needs clearly defined requirements. This section of the framework will include:

- Intended Use

- Data and Metadata Requirements

- Quality

- Provenance

- Timeliness

- Management

- Interoperability

- Security

- Functional Requirements

- Interactions with other MSA capabilities

- User Interface Requirements

- Performance Requirements

- Credibility and Evaluation Requirements

- Theoretical corroboration

- Model components verification

- Corroboration (independent data)

- Sensitivity analysis

- Uncertainty analysis 
- Robustness determination

- Comparison to evaluation criteria

Examples of functional requirements for HMR are provided below.

- Predict the initial direction, travel, and dispersion of a plume over time from a single or multiple sources taking into account the type of source, material/chemical properties, release location, weather conditions, terrain, urban areas, and other man-made structures.

- Predict the concentration of the chemical or biological agent within the plume and flow through drainage areas over time.

- Estimate deposition and contamination levels for air, water, ground, and building surfaces.

- Identify exposed population and predict exposure levels over time.

\section{IDENTIFICATION OF EXISTING MSA RESOURCES}

This section of the framework will capture MSA resources available for systems analyses for the technical area. These are categorized into the following sub-sections.

\subsection{Projects, Facilities, and Capabilities}

The sub-section will identify the ongoing projects, facilities, and capabilities focused on MSA the technical area. Examples for the CI area include:

National Infrastructure Simulation and Analysis Center (NISAC): NISAC is a modeling, simulation, and analysis program within DHS comprising personnel in the Washington, D.C., area as well as from Sandia National Laboratories (Sandia) and Los Alamos National Laboratory (LANL). A facility dedicated to NISAC is located at Sandia Albuquerque. Congress mandated that NISAC serve as a "source of national expertise to address critical infrastructure protection research and analysis." NISAC prepares and shares analyses of critical infrastructure and key resources (CIKR), including their interdependencies, vulnerabilities, consequences, and other complexities, under the direction of the Office of Infrastructure Protection (IP), Infrastructure Analysis and Strategy Division (IASD). As of September 2008, NISAC had conducted and published 11 analyses of hurricanes covering the entire U.S. Gulf and Atlantic coast (USDHS 2010b).

Chemical Sector Supply Chain and Economics Project: This project is a key component of a larger effort to deliver Enabling Homeland Security Capabilities (EHCs) for the Modeling, Mapping, and Simulation program. The first goal of this project is to populate a detailed dataset of the chemical and petrochemical manufacturing, supply and distribution components that comprise the chemical infrastructure supply chain. The second goal is to develop a means to mathematically analyze not only the consequence of significant threats, but also the resiliency of the supply chain to recover from these impacts. This project is part of the Critical Infrastructure Protection Thrust Area and the Modeling, Simulation \& Analysis Program of the Infrastructure and Geophysical Division of DHS.

Ongoing projects will also include efforts in academia as described in research literature. For example, recent reported efforts on infrastructure simulations in academia include energy distribution (Baxevanos and Labridis 2007) and water supply (Qiao et al. 2007).

Examples for the HMR area include:

National Atmospheric Release Advisory Capability (NARAC) - The NARAC facility is located at Lawrence Livermore National Laboratory in Livermore, CA. It provides tools and expert services to map the spread of hazardous material accidentally or intentionally released into the atmosphere (NARAC 2010).

EPA's National Exposure Research Lab (NERL) - located in Research Triangle Park, North Carolina provides scientific understanding, information and assessment tools to reduce and quantify the uncertainty in the Agency's exposure and risk assessments for all environmental stressors. The Atmospheric Sciences 
Modeling Division provides numerical and physical modeling support to the homeland security mission in protecting against the environmental and health effects of terrorist acts (EPA 2010). This involves numerical modeling complemented by physical modeling in the Division's wind tunnel. For example, a 1:600 scale model of lower Manhattan was built and the dispersion of material from the collapse of the World Trade Center towers was studied under various meteorological conditions. Also, dispersion of airborne material around the Pentagon was simulated in the wind tunnel.

\subsection{MSA Tools Summary}

This sub-section will provide a collection of available MSA tools (the tools under development are identified in the preceding sub-section as projects). The tools will be briefly described to provide an overview of their capabilities. In the long term, this information should be enhanced to grow the collection to the compendium of models called for in the workshop focused on CI (Adam 2008). The brief tool descriptions will provide a useful resource until the research issues related to the proposed compendium are addressed. An interested reader can use the description to quickly develop a shortlist of tools that may be applicable for their purpose and can then follow-up to find more details. Examples of such descriptions of MSA tools for CI area are provided below.

Critical Infrastructure Protection Decision Support System (CIPDSS): Developed jointly by LANL, Sandia and Argonne National Labs (ANL) the set of tools under the CIPDSS program models the impact of CI on the economy, government, and population. LANL developed the city level models, Sandia developed the national level models while ANL provided the decision support part. The set of tools is intended to provide "orders of magnitude" results quickly. It was used for the analysis underlying NISAC's report on potential impact of pandemic influenza (NISAC 2007).

Critical Infrastructure Protection and Resiliency Simulator (CIPR/sim): Developed by Idaho National Labs (INL) CIPR/sim allows emergency planners to visualize the real-time cascading effects of multiple infrastructure failures before an actual emergency occurs. It uses a common operating framework that allows the tool to import real-time data from numerous existing analysis modules, including RTDS (Real Time Digital Simulator) for electric grid analysis, QualNet for telecommunications analysis, and PC Tide for wind speed and flood surge analysis (INL 2010).

Examples of MSA tools for healthcare systems area include the following.

EpiSimS. The epidemic simulation engine, EpiSimS is a $\mathrm{C}++$ application that runs on highperformance computing clusters. It is a stochastic agent-based discrete event model that explicitly represents every person in a city, and every place within the city where people interact. A city or region is represented physically by a set of road segment locations and a set of business locations. EpiSimS can simulate various pharmaceutical and non-pharmaceutical interventions, including panic-based stay-home behavior, therapeutic and prophylactic use of antivirals, contact tracing, vaccination, wearing of masks, social distancing behaviors (increased inter-personal separation, hand washing, cough etiquette, etc.), household quarantine, and closures of schools. More information is available in Stroud et al. (2007).

MedModel. MedModel is a simulation tool designed specifically for the healthcare industry. MedModel is used in the evaluation, planning and redesign of hospitals, clinics, and other healthcare systems. In the hands of a trained and experienced analyst, MedModel models can be used to identify inefficiencies in an existing process and test a variety of scenarios. The animation and graphic output results show the behavior of the system under any set of circumstances (Promodel 2010).

\subsection{Relevant Standards and Guidelines}

This sub-section will identify the known available relevant standards and guidelines. A few examples for this sub-section for CI area are provided below.

National Infrastructure Protection Plan (NIPP): NIPP is a coordinated strategy that defines CIKR protection roles and responsibilities for federal, state, local, tribal, and private sector security partners. The NIPP sets national priorities, goals, and requirements for effective distribution of resources which 
will enable the government, economy, and public services to continue in the event of a terrorist attack or other disaster.

Sector-Specific Plans (SSPs): SSPs have been developed for each of the identified CI sectors supporting the NIPP.

Chemical Facility Anti-Terrorism Standards (CFATS): CFATS is a regulatory program to secure national high-risk chemical facilities. Over 30,000 potentially high-risk chemical facilities have already completed consequence assessments under this program as of September 2008 (USDHS 2010b).

Examples of standards and guidelines for healthcare systems include:

Smallpox Response Plan \& Guidelines. This document from Centers for Disease Control and Prevention (CDC) outlines the public health strategies that would guide the public health response to a smallpox emergency and many of the federal, state, and local public health activities that must be undertaken in a smallpox outbreak.

Community-Based Mass Prophylaxis: A Planning Guide for Public Health Preparedness. This is a planning guide developed by Agency of Healthcare Research \& Quality (AHRQ) to help state, county, and local officials meet federal requirements to prepare for public health emergencies. It outlines five components of mass prophylaxis response to epidemic outbreaks and addresses dispensing operations using a comprehensive operational structure for Dispensing/Vaccination Centers (DVCs) based on the National Incident Management System (NIMS).

\subsection{Data Sources and Formats}

The sources of data relevant to MSA technical areas and their identified formats will be provided in this sub-section. An example for CI area is as below.

Constellation/Automated Critical Asset Management System (C/ACAMS): A web-enabled information services portal that helps state and local governments build critical protection programs in their local jurisdictions. ACAMS is a secure, online database, and database management platform that allows for the collection and management of CI asset data; the cataloguing, screening, and sorting of this data; the production of tailored infrastructure reports; and the development of a variety of pre- and post-incident response plans useful to strategic and operational planners and tactical commanders.

The data sources for healthcare systems area may include the following example.

National Emergency Medical Services (EMS) Information System (NEMSIS). The system is the national repository that will be used to potentially store EMS data from every state in the nation. Since the 1970s, the need for EMS information systems and databases has been well established, and many statewide data systems have been created. The involved organizations include National Highway Traffic Safety Administration (NHTSA), Health Resources and Services Administration (HRSA), CDC, University of Utah, and University of North Carolina.

\section{DISCUSSIONS AND RECOMMENDATIONS}

The discussion and recommendations section in the framework will include identified best practices, limitations, cautions, and warnings, and research, development, standards and implementation issues. Recommendations can then be considered by government organizations for funding and execution.

\subsection{Best Practices for Development and Use of MSA Tools}

The development and use of MSA tools can benefit through development and use of lessons learned and sharing of best practices. This sub-section would document and capture lessons learned and best practices acquired in development, evaluation, and use of MSA tools for the technical area. Some examples of best practices include:

- Conceptual modeling practice

- Innovative approaches 
- Software engineering practice

\subsection{Limitations, Cautions, and Warnings}

Models provide results with varying levels of error and uncertainty. This sub-section is intended to highlight and document the limitations associated with MSA applications to minimize improper use and highlight potential areas for further development.

\subsection{Research, Development, Standards and Implementation Issues}

This section of the framework is intended to capture challenges and issues related to MSA tools in the categories defined by the following sub-sections. The challenges and issues should be prioritized for maximum value.

\subsubsection{Research and Development}

A number of research and development challenges need to be addressed in modeling potential threats for the technical area and impact of disruptions due to involved phenomena. Common challenges across the technical areas should be identified such as the development of simulation application architectures and integration of models and simulations for the technical area. In addition, challenges need to be identified and prioritized for each technical area. For example for CI area, computational modeling to understand the vulnerability of a dam to explosions would be useful in mitigating this threat. Physical models are necessary to collect data on impact of explosions to support evaluation of the modeling capability to ensure credibility of result. However, a physical model of a dam cannot be scaled down to laboratory settings since the physics changes based on the scale. Hence, expensive large scale experiments may be required for the purpose. An initial straw man list of such challenges for the healthcare systems area may include, increasing reality in healthcare M\&S training exercises and devices, access to and usage of healthcare M\&S applications by system personnel.

\subsubsection{Standards}

The success of a coordinated approach will largely depend on the ability to apply data-driven MSA tools across a range of scenarios. This ability in turn depends on use of standards. Gaps in available standards should be identified and prioritized to guide standardization efforts. At times the issue may be to identify a common standard from among multiple ones available. For example, issues with multiple Geographic Information System (GIS) standards should be identified and an approach to identify one common GIS standard to be followed for outputs by all MSA tools for a particular area should be identified or developed.

\subsubsection{Implementation}

Deployment of new MSA tools for homeland security has to follow a carefully organized approach. The approach may vary across the technical areas depending on the state of technology, the familiarity of the end users with MSA, and the interfaces with the end users. Common implementation challenges across the technical areas include return on investment to stakeholders and sponsors for research projects, and ownership and usage of publicly versus privately developed tools. For example, the eighteen identified CI domains involve a myriad of stakeholders including government, quasi-government, and private sector operators, a number of oversight agencies across federal, state, and local levels, a range of jurisdictions involved in normal operation, and another range of jurisdiction that may be affected under disrupted or interrupted operations. Some sectors may require use of "reachback" centers, i.e., entities with expertise available to guide the users in interpretation of results. Other sectors may have sophisticated users who can be trained to use the MSA tools independently. 


\section{FRAMEWORK REFERENCE MATERIALS}

The knowledge sharing framework will need to be supported by a number of reference materials provided via appendices. While every attempt should be made to use standard terminology, more often than not, this may not be possible due to its absence. The technical interest group should guide the generation of standard terminology that should be defined in a glossary.

The framework is expected to include technical discussions and build on existing current literature. A reference section should be included to capture the relevant publications.

Potential and existing users of MSA tools may need guidance from experts in respective areas. A list of identified experts for each major aspect of each technical area should be captured. The list may be restricted to authorized personnel and the names on the list may be included only with permission. The experts may need support for the time they may have to spend fielding questions.

\section{CONCLUSION}

This paper describes a knowledge sharing framework for MSA for homeland security. It is suggested that the wide area of homeland security be divided into several technical areas based on existing communities of researchers. The knowledge sharing framework can then be used to pull together and create an information source for each technical area. The collection and maintenance of information, identification of research challenges, and development of the research agenda should be coordinated by technical interest groups. Establishing such a framework for MSA capabilities is one approach to encourage collaboration and coordination for systems analysis, problem solving, and decision making for each of the technical areas. The framework should be documented and made available to stakeholders as an on-line resource such as a secure portal or Wiki to enable development and progress of MSA for homeland security purposes.

The framework outlined in Sections 2 through 7 is applicable across multiple domains as indicated by the examples used from critical infrastructure, hazardous material release, and healthcare systems technical areas.

\section{ACKNOWLEDGMENTS}

The Science and Technology Directorate of the U.S. Department of Homeland Security sponsored the production of this material under Interagency Agreement HSHQDC-08-X-00418 with the National Institute of Standards and Technology (NIST). NIST sponsored a part of this work to the George Washington University under grant number 707NANB8H8167. The work described was funded by the United States Government and is not subject to copyright.

\section{DISCLAIMER}

Some software products or services may have been identified in the context in this paper. This does not imply a recommendation or endorsement of such products or services by the authors, NIST, or DHS; nor does it imply that such products or services are necessarily the best available for the purpose.

\section{REFERENCES}

Adam, N. 2008. Workshop on Future Directions in Critical Infrastructure Modeling and Simulation, Final Report. U. S. Department of Homeland Security, Infrastructure \& Geophysical Division, Science and Technology Directorate, Washington, DC.

Baxevanos, L.S., and D. P. Labridis. 2007. Implementing Multiagent Systems Technology for Power Distribution Network Control and Protection Management. IEEE Transactions on Power Delivery, 22(1):433-443. 
Brase, J.M., and D.L. Brown. 2009. Modeling, Simulation and Analysis of Complex Networked Systems: A Program Plan. Livermore, CA: Lawrence Livermore National Laboratory. Available via <https://wiki.cac.washington. edu/download/attachments/7478403/Comple xNetworkedSystems Program-final.pdf?version=1> [accessed February 08, 2010].

DoD DCIO 2010. Department of Defense Architecture Framework (DoDAF) Version 2.0. DoD Deputy Chief Information Officer. Available via: <http://cionii.defense.gov/sites/dodaf20/> [accessed August 16, 2010].

EPA 2010. Atmospheric Modeling and Analysis Division. U.S. Environmental Protection Agency. Available via: <http: //www.epa.gov/asmdnerl/> [accessed August 16, 2010].

Hutchings, C.W. 2009. Enabling Homeland Security with Modeling \& Simulation (M\&S). Interservice/Industry Training, Simulation, and Education Conference (I/ITSEC), Paper ID\# 9512, Orlando, FL, Nov. 30-Dec. 3.

INL 2010. Research Fact Sheet: Critical Infrastructure Resiliency Simulation (CIPR/Sim). Idaho National Laboratory. Available via: <http://www.inl.gov/research/criticalinfrastructure-resiliency-simulation/> [accessed August 16, 2010].

McLean, C. R., S. Jain, and Y. T. Lee. 2008. A Taxonomy of Homeland Security Modeling, Simulation, and Analysis Applications. Simulation Interoperability Workshop (SIW), Paper No. 08S-SIW-098, Providence, RI, April 14-18.

NARAC (2010). National Atmospheric Release Advisory Center. Available via: <https://narac.llnl.gov> [accessed August 16, 2010].

NISAC 2007. National Population, Economic, and Infrastructure Impacts of Pandemic Influenza with Strategic Recommendations. National Infrastructure Simulation \& Analysis Center, Infrastructure Analysis and Strategy Division, Office of Infrastructure Protection, U.S. Department of Homeland Security.

ProModel Corporation 2010. MedModel - The Industry Standard for Healthcare Simulations. ProModel Corporation. Available via: <http://www.promodel.com/products/medmodel> [accessed August 16, 2010].

Qiao, J., D. Jeong, M. Lawley, J. P. Richard, D. M. Abraham, and Y. Yih. 2007. Allocating Security Resources to a Water Supply Network. IIE Transactions, 39(1):95-109.

Stroud P.D., S.Y. Del Valle, S.J. Sydoriak, J.M. Riese, and S.M. Mniszewski. 2007. Spatial Dynamics of Pandemic Influenza in a Massive Artificial Society. Journal of Artificial Societies and Social Simulation 10(4):9. Available via: <http://jasss.soc.surrey.ac.uk/10/4/9.html> [accessed July 21, 2010]

USDHS, 2010a. Critical Infrastructure and Key Resources, 2010. U. S Department of Homeland Security. Available via <http://www.dhs.gov/files/programs/gc_1189168948944.shtm> [accessed January 12, 2010].

USDHS, 2010b. Fact Sheet: Critical Infrastructure and Homeland Security Protection Accomplishments, 2010. U. S. Department of Homeland Security. Available via <http://www.dhs.gov/xnews/releases/pr_1220878057557.shtm> [accessed January 14,2010$]$.

John A. Zachman, 2008. The Zachman Framework ${ }^{T M}$ : The Official Concise Definition. Available via: <http://www. zachmaninternational.com>. [accessed August 16, 2010].

\section{AUTHOR BIOGRAPHIES}

SANJAY JAIN is an Associate Professor in the Department of Decision Sciences, School of Business at the George Washington University. Before moving to academia, he accumulated over a dozen years of industrial R\&D and consulting experience working at Accenture in Reston, VA, USA, Singapore Institute of Manufacturing Technology, Singapore and General Motors North American Operations Technical 
Center in Warren, MI, USA. He received a Bachelors of Engineering from Indian Institute of Technology (IIT)-Roorkee, India, a Post Graduate Diploma from National Institute of Industrial Engineering, Mumbai, India, and a Ph.D. in Engineering Science from Rensselaer Polytechnic Institute, Troy, New York. $\mathrm{He}$ is an associate editor of the International Journal of Simulation and Process Modelling and a member of the editorial board of the International Journal of Industrial Engineering. His research interests are in application of modeling and simulation to complex scenarios including homeland security and supply chains. His web-page address is <http://business.gwu.edu/faculty/sanjay_jain.cfm> and his email address is <jainegwu.edu>.

CHARLES W. HUTCHINGS is Deputy Director, Modeling and Simulation (M\&S) in the Test\& Evaluation and Standards Division of the Science \& Technology Directorate, U.S. Department of Homeland Security, and is responsible for providing modeling, simulation, and analysis support and guidance to the Directorate and associated programs to promote the advancement and judicious use of M\&S. Before transferring to DHS in November 2007, Dr. Hutchings was the Senior Analyst in the Navy Test, Evaluation, and Technology Division where he provided subject matter expertise in M\&S for test and evaluation (T\&E) and led a program to develop an alternative approach to full ship shock trials based on M\&S. Dr. Hutchings has ten years experience supporting Navy programs. Prior to government service, Dr. Hutchings completed post-doctoral work and was appointed Visiting Professor in the Department of Physics at the University of Nebraska-Lincoln and authored/co-authored 17 papers in condensed matter physics and surface science. He received a Ph.D. in Physical Chemistry from the University of Heidelberg, Heidelberg, Germany in 1994, and received a M.S. in Physics and B.S. with majors in Mathematics and Physics from Syracuse University in Syracuse, NY in 1990 and 1987, respectively. His email address is $<$ Charles.Hutchings@dhs.gov>.

Y. TINA LEE is a computer scientist and acting group leader of the Manufacturing Simulation and Modeling Group at the National Institute of Standards and Technology (NIST). She joined NIST in 1986. Her major responsibility in recent years has been to develop information models to support various manufacturing applications. She is currently the secretary of the Core Manufacturing Simulation Data (CMSD) Product Develop Group of the Simulation Interoperability Standards Organization (SISO) and co-editor of the CMSD specification. She received her BS in Mathematics from Providence College and MS in Applied Science from the College of William and Mary. Her e-mail address is leet@nist.gov>.

CHARLES R. MCLEAN is a computer scientist and retired Group Leader of the Manufacturing Simulation and Modeling Group at the National Institute of Standards and Technology. He has managed research programs in manufacturing simulation, engineering tool integration, product data standards, and manufacturing automation at NIST since 1982. He has authored more than 50 technical papers on topics in these areas. He has served on the Executive Board of the Winter Simulation Conference and the Editorial Board of the International Journal of Production, Planning, and Control. He is formerly the Vice Chairman of the International Federation of Information Processing (IFIP) Working Group on Production Management Systems (WG 5.7). He holds an M.S. in Information Engineering from University of Illinois at Chicago and a B.A. from Cornell University. His e-mail address is <charles mcleanenist.gov>. 